\title{
Influence du type de station forestière sur les composantes intracernes de la densité du bois du chêne pédonculé (Quercus robur $L$ ) dans les chênaies de l'Adour et des coteaux basco-béarnais
}

\author{
F Ackermann \\ CEMAGREF, groupement de Bordeaux, division Production et Économie agricoles, \\ 50, avenue de Verdun, BP 3, 33611 Gazinet cedex, France
}

(Reçu le 24 mai 1994 ; accepté le 8 juin 1995)

\begin{abstract}
Résumé - En aval d'une typologie des stations forestières, 3 grands types de stations, bien distincts, où le chêne pédonculé est largement représenté, ont été choisis pour étudier l'influence des facteurs stationnels sur les composantes intracernes de la densité du bois (densité moyenne du cerne, densités du bois initial et du bois final) considérées comme des critères de qualité interne du bois de cette essence. Ces paramètres ont été recueillis par voie non destructive à partir de l'analyse microdensitométrique de carottes de sondage, prélevées à cœur sur 105 arbres, sélectionnés au nombre de 5 dans 7 placettes par type de station. Cette analyse a également fourni, pour les 8793 cernes mesurés, leur largeur, les largeurs de leur bois initial et de leur bois final, ainsi que leur âge compté depuis la moelle. Toutes ces données ont fait l'objet d'une analyse de variance à 3 critères de classification hiérarchisés (type de station, placette dans type de station, arbre dans placette). Les résultats ont montré qu'à âge de cerne fixé puis qu'à âge et largeur de cerne fixés l'essentiel de la variabilité observée, pour les 3 composantes intracernes de la densité du bois, était imputable au facteur individuel («effet arbre»). Mais, malgré cela, ces caractéristiques apparaissent significativement influencées par le type de station. Cette relation entre le type de station et les composantes microdensitométriques des accroissements annuels pourrait s'expliquer par une action des facteurs du milieu sur les caractéristiques anatomiques des cernes.
\end{abstract}

type de station / qualité du bois / microdensitométrie / densité du bois / chêne pédonculé

Summary - Relationship between forest site and intra-ring wood density components for the pedunculate oak (Quercus robur $\mathrm{L}$ ) of southwestern France. After a forest site classification, 3 forest sites characterised by distinct ecological conditions, where the pedunculate oak is largely present, have been selected to study site factors' influence on the intra-ring wood density components (ring 
average density, earlywood and latewood density) considered as wood quality criteria for this species. These parameters have been collected by a nondestructive mean based on the microdensitometric analysis of increment cores extracted from 105 trees chosen according to a 3-stage sampling (3 sites, 7 stands into site, 3 trees into stand). This analysis has also provided the total width, the width of the earlywood and latewood and age (position from the pith) of the 8793 rings studied. These data have been treated by a 3 hierarchised classification factors (site, stand into site, tree into stand) variance anal$y$ sis. The results have shown that, when cambial age is fixed and when cambial age and ring width are fixed, the variation of wood density parameters result, in large part, from an important intertree variability. However, forest site influence on wood density parameters is significant and it seems that site factors act on the anatomic wood characteristics.

\section{forest site / wood quality / microdensitometry / wood density / pedunculate oak}

\section{INTRODUCTION}

Les typologies des stations forestières ont pour objectif d'élaborer un outil pratique (le catalogue des types de stations) permettant au forestier d'individualiser des surfaces homogènes du point de vue des conditions écologiques et donc des potentialités forestières.

Ce concept de potentialité forestière est le plus souvent abordé, pour une essence donnée, en termes de productivité qui exprime la quantité de matière ligneuse produite par unité de surface et de temps. Aussi est-il assez fréquent d'étudier, en aval des typologies, les relations station-production pour diverses essences (Becker et al, 1980 ; Le Goff et Madesclaire, 1985 ; Madesclaire, 1986 ; Franc et Curt, 1990 ; Farrinotti et Larousse, 1991 ; Gilbert et al, 1991).

Néanmoins, sur le plan économique, il apparaît que, pour de nombreuses essences et en particulier pour le chêne, les qualités externe et interne de la matière ligneuse produite prévalent sur la quantité (Polge et Keller, 1973 ; Normandin, 1990 ; Polge, 1990). C'est pourquoi, le CEMAGREF de Bordeaux, après avoir été sollicité pour réaliser une typologie des stations forestières (Ackermann, 1994a) dans une région où le chêne pédonculé est omniprésent (fig 1), a jugé opportun d'étudier parallèlement l'influence du type de station forestière sur la qualité du bois de cette essence.
Ce thème de recherche, qui a fait l'objet d'une thèse au sein de cet établissement (Ackermann, 1994b), avait déjà été abordé par de nombreux auteurs (Zahner, 1968 ; Le Tacon et Mormiche, 1974 ; Polge, 1973, 1975 ; Courtoisier, 1976 ; Pellecuer, 1976 ; Becker, 1979 ; Mourey, 1979 ; Drapier, 1987 ; Klumpers, 1990).

Cependant les conclusions de ces diverses études, portant sur la recherche d'une éventuelle influence des facteurs du milieu (ou du type de station) sur les propriétés du bois du chêne sessile (Quercus petraea Liebl) et du chêne pédonculé (Quercus robur $L$ ), restent mitigées et parfois contradictoires.

En effet, il est apparu très difficile de discerner, dans la variabilité des caractères du bois mesurés, la part revenant à l'influence des facteurs du milieu de celle générée par 2 grands types de facteurs :

- les facteurs génétiques qui constituent une source de variation interspécifique mais également intraspécifique : variabilité interprovenance qui induit une variabilité interpeuplement (Nepveu, 1990) et très forte variabilité génétique individuelle au sein d'une même provenance conduisant à la grande variabilité observée dans les peuplements (Nepveu, 1990, 1993) ;

- les facteurs sylvicoles au sens large. Ils regroupent la vitesse de croissance et l'âge des arbres. Des études récentes de l'équipe de recherches sur la qualité des bois 


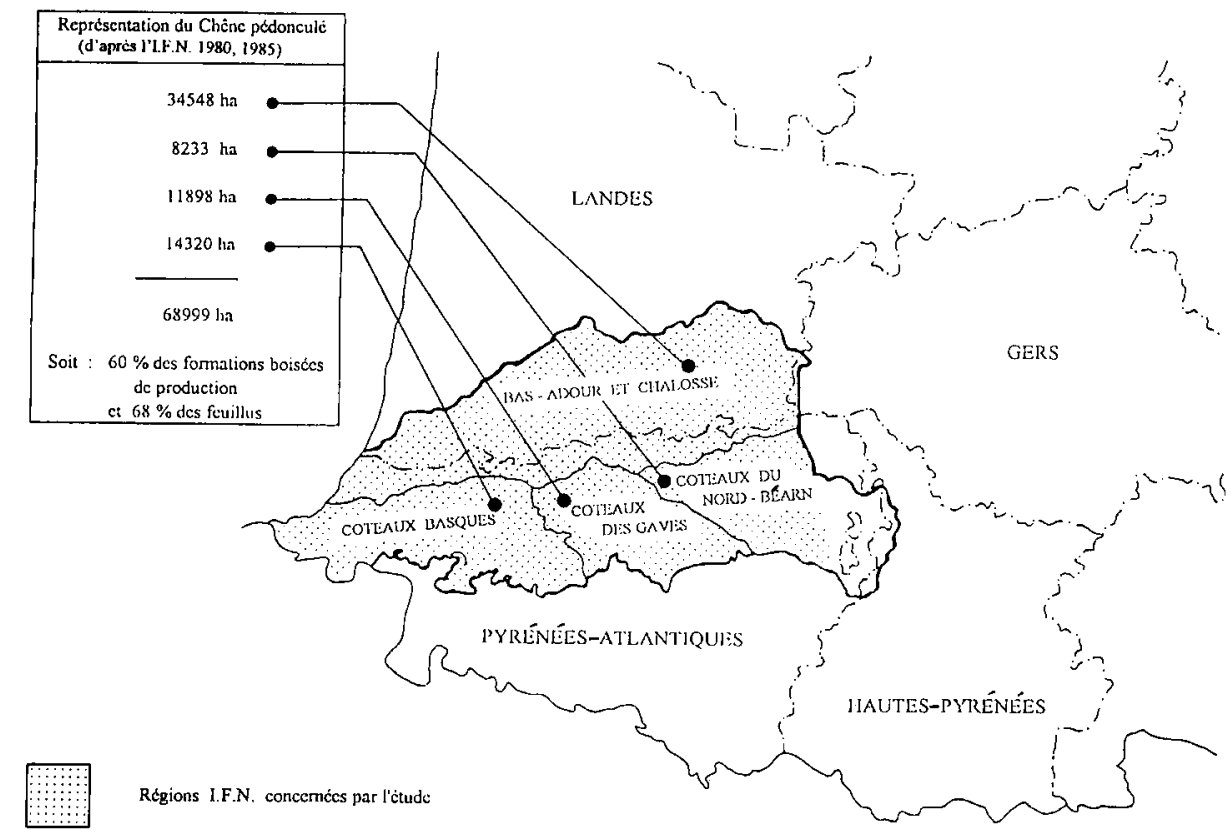

Fig 1. Représentation du chêne pédonculé dans les formations boisées de production des régions forestières étudiées.

(ERQB) de I'INRA-Nancy et en particulier les travaux d'Eyono Owoundi (1992), poursuivis par Zhang et al (1993), ont montré que la largeur de cerne et, indépendamment de celle-ci, l'âge des accroissements annuels (âge compté depuis la moelle) influençaient considérablement les propriétés du bois. Ces facteurs constituent donc eux aussi une source de variabilité intra et inter-peuplement déjà mise en évidence par Polge et Keller (1973).

Les objectifs de notre étude étaient donc les suivants :

- mettre au point un protocole qui puisse rendre compte de la seule influence des facteurs stationnels sur la qualité intrinsèque du bois. Nous verrons que ce protocole repose sur une échelle de mesure, un plan d'échantillonnage et des traitements statistiques permettant de contrôler, en plus du type de station, les autres facteurs influen- çant fortement les propriétés du bois (vitesse de croissance et âge des accroissements annuels) ;

- appliquer ce protocole à titre exploratoire sur un certain nombre de types de stations afin d'apporter quelques éléments de réponse sur la relation station-critères de qualité du bois pour le chêne pédonculé.

Cette démarche présente un intérêt scientifique incontestable puisqu'au début de l'étude on ne pouvait pas se prononcer sérieusement sur une quelconque influence des facteurs stationnels (qui ne passe pas par la vitesse de croissance) sur les propriétés intrinsèques du bois pour le chêne (Nepveu, 1992).

De plus, dans la région étudiée, le fait de savoir s'il existe des stations plus ou moins favorables à la production de bois de chêne de qualité est de toute première importance sur le plan économique et sylvicole. En effet, 
le maintien du chêne pédonculé, dans de nombreux peuplements, est déjà remis en question en raison de la médiocre qualité des produits récoltés (fort taux de gélivures notamment).

Dans cet article, nous développerons le protocole utilisé et exploiterons les résultats issus d'analyses de variance à plusieurs critères de classification hiérarchisés (type de station, placette dans le type de station, arbre dans la placette) réalisées sur diverses caractéristiques intrinsèques du bois (composantes intracernes de la densité du bois et paramètres relatifs à la structure des cernes) mesurées sur un premier échantillon de 105 chênes pédonculés.

\section{MATÉRIEL ET MÉTHODES}

\section{Échelle d'observation et paramètres d'estimation de la qualité du bois}

Ėtant donné la très forte variabilité individuelle des propriétés du bois observée chez le chêne, il est nécessaire, dans le cadre d'une étude des relations station-qualité du bois, d'échantillonner un grand nombre d'arbres. La taille de l'échantillon justifie donc le choix d'une méthode non destructive (Nepveu, 1988, $1989 ; 1990$, 1992).

Puisque l'on s'intéresse à la qualité interne du bois, le matériel d'expérience est constitué de carottes de sondage prélevées à $1,3 \mathrm{~m}$ dans la bille de pied.

Comme dans la pratique il était impossible d'échantillonner a priori des chênes de peuplements différents présentant des âges et des vitesses de croissance similaires, les carottages ont été effectués à cœur et les critères d'estimation de la qualité du bois ont été mesurés à l'échelle de l'accroissement annuel afin de pouvoir contrôler par la suite ces 2 facteurs influençant fortement les propriétés internes du bois.

À cette échelle, l'estimation de la qualité du bois peut être réalisée de façon fiable et précise par la mesure des caractéristiques densitométriques. Celles-ci sont en effet très bien corrélées à un grand nombre de propriétés technologiques (en particulier la rétractibilité) qu'il serait fastidieux, voire impossible, de mesurer sur ce type d'échantillon (Polge, 1966 ; Nepveu, 1989 ; Zhang et al, 1994).

\section{Stratégie d'échantillonnage}

\section{Choix des types de stations}

Les types de stations sur lesquels l'étude a porté ont été choisis en nombre limité pour des raisons matérielles et du fait du caractère exploratoire de l'étude.

II s'agit de types de stations où le chêne pédonculé est bien représenté et qui se différencient bien entre eux du point de vue des exigences autoécologiques de l'essence.

La figure 2 replace les 3 types de stations choisis par rapport à la distribution stationnelle régionale du chêne pédonculé et à ses conditions écologiques optimales de croissance (Ackermann, 1994a, b).

Ainsi, le premier type de station (type 1) correspond aux conditions écologiques optimales de l'essence. Il s'agit de stations neutrophiles hygroclines de vallée.

Le second type de station (type 2) se différencie du premier par un niveau hydrique beaucoup moins favorable à l'essence. Il correspond aux stations neutrophiles à mésoneutrophiles, mésophiles, de coteaux.

Le troisième type de station (type 3) présente des conditions trophique et hydrique défavorables au chêne pédonculé. II s'agit de stations acidiphiles à acidiphiles modérées, mésophiles, de coteaux.

\section{Plan d'échantillonnage}

Le plan d'échantillonnage adopté correspond à un modèle hiérarchique à 3 niveaux de classification : le type de station, la placette à l'intérieur du type de station et l'arbre à l'intérieur de la placette.

II doit permettre de distinguer la variabilité inter-type de station (celle qui nous intéresse) par rapport à la variabilité intra-type de station, due à la variabilité inter-placette (ou inter-peuplement), dans laquelle se trouve emboîtée la variabilité intra-placette (variabilité individuelle). La 
variabilité résiduelle correspond à la variabilité entre les cernes à l'intérieur de l'arbre.

Pour chacun des 3 types de stations, 7 placettes ont été choisies dans des peuplements adultes, gérés par l'Office national des forêts, présentant des structures semblables (futaie claire) et ne posant pas, a priori, de problème de provenance.

Leur superficie n'a été fonction que de l'homogénéité stationnelle.

Leur nombre ( 7 par type de station) était le nombre maximal que l'on pouvait atteindre sans alourdir considérablement la phase de prospection.

À l'intérieur de ces placettes, 5 arbres ont été échantillonnés. Ils devaient avoir un statut d'arbre dominant ou codominant et ne pas présenter, autant que faire se peut, de défauts majeurs susceptibles de perturber leur plan ligneux (fentes ou gélivures franches, courbure ou inclinaison marquée).
Le nombre de 5 arbres par placette correspondait à l'effectif optimal qu'il était possible d'obtenir en respectant les critères de sélection fixés et l'homogénéité stationnelle.

Chaque type de station est donc représenté par 35 arbres répartis sur 7 placettes. Ceci correspond à un échantillon global de 105 arbres sur lesquels le carottage a été effectué sans direction privilégiée mais en évitant toute zone de bois de tension.

\section{Techniques de mesures}

Les carottes de sondage, après avoir été redressées sous presse et stabilisées à $12 \%$ d'humidité, ont été sciées, perpendiculairement au fil du bois, en planchettes de $2 \mathrm{~mm}$ d'épaisseur, à l'aide de scies-fraises jumelées (dispositif décrit par Perrin, 1983). Celles-ci ont ensuite été radiographiées suivant les conditions d'irradiation suivantes : distance source-film : $2,50 \mathrm{~m}$, tension
Fig 2. Situation des types de stations étudiés par rapport à la distribution stationnelle régionale du chêne pédonculé et aux conditions écologiques optimales de croissance de cette essence.

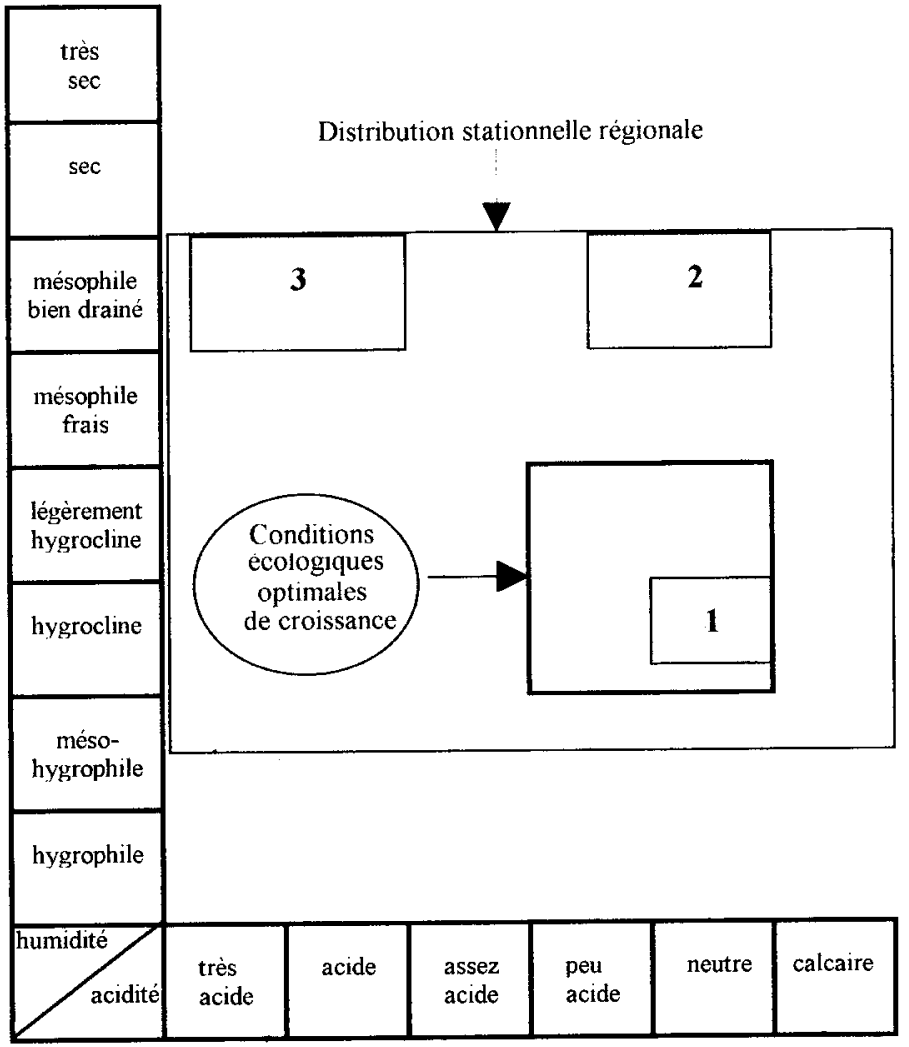


accélératrice : $10 \mathrm{KV}$, intensité du flux : $10 \mathrm{~mA}$, temps d'exposition : $4 \mathrm{~h}$.

Les radiographies ainsi obtenues ont été analysées au moyen d'un microdensitomètre enregistreur (dernier modèle de I'ERQB). Chaque carotte a été parcourue 3 fois par le faisceau, de la moelle vers l'écorce. Les axes des 3 passages parallèles étaient distants de $700 \mu \mathrm{m}$. La hauteur de la fenêtre de mesure était fixée à $500 \mu \mathrm{m}$ et la largeur à $25 \mu \mathrm{m}$. Le pas de mesure était de 24 $\mu \mathrm{m}$.

Les données enregistrées ont été traitées par un programme d'analyse spatiale permettant de fixer la position des limites de cernes et de calculer, entre autres, pour chaque cerne et pour chacun des passages, les données synthétiques suivantes, exploitées dans cet article :

- l'âge cambial (âge compté depuis la moelle), obtenu grâce à la saisie de l'âge estimé du premier cerne disponible sur la carotte ;

- la largeur totale (LC), la largeur du bois initial (LBI) et du bois final (LBF) qui caractérisent la structure du cerne ;

- la densité moyenne dans le cerne (Dmoy), la densité du bois initial (DBl) et du bois final (DBF);

- l'angle d'inclinaison de la limite de cerne calculé en fonction des coordonnées des mesures effectuées sur les 3 passages, qui sert postérieurement à corriger les largeurs.

Tableau I. Moyennes et écarts types de l'âge des arbres sondés, nombre de cernes mesurés et moyennes et écarts types de la largeur des cernes par placette et par type de station.

\begin{tabular}{|c|c|c|c|c|c|c|}
\hline \multirow[t]{2}{*}{ Type de station } & \multirow[t]{2}{*}{ Placette } & \multicolumn{2}{|c|}{ Âge des arbres } & \multirow[t]{2}{*}{$\begin{array}{c}\text { Nombre de } \\
\text { cernes mesurés }\end{array}$} & \multicolumn{2}{|c|}{$\begin{array}{l}\text { Largeur de } \\
\text { cerne }(\mathrm{mm})\end{array}$} \\
\hline & & $\mathrm{m}$ & $\sigma$ & & $m$ & $\sigma$ \\
\hline & 1 & 60,0 & 1,8 & 280 & 2,68 & 1,71 \\
\hline & 2 & 90,2 & 1,1 & 396 & 2,80 & 1,39 \\
\hline & 3 & 89,2 & 2,5 & 402 & 3,31 & 1,57 \\
\hline \multirow[t]{8}{*}{1} & 4 & 100,2 & 3,7 & 444 & 2,62 & 1,34 \\
\hline & 5 & 62,8 & 2,6 & 286 & 2,87 & 1,66 \\
\hline & 6 & 70,0 & 5,4 & 309 & 3,39 & 1,96 \\
\hline & 7 & 60,0 & 3,7 & 264 & 4,82 & 2,18 \\
\hline & Total & 76,1 & 15,8 & 2381 & 3,15 & 1,79 \\
\hline & 1 & 104,0 & 17,0 & 457 & 2,70 & 1,64 \\
\hline & 2 & 65,2 & 3,7 & 280 & 2,46 & 1,23 \\
\hline & 3 & 121,4 & 1,5 & 552 & 1,61 & 0,87 \\
\hline \multirow[t]{8}{*}{2} & 4 & 90,0 & 12,4 & 409 & 2,71 & 1,32 \\
\hline & 5 & 91,2 & 9,1 & 408 & 3,12 & 1,36 \\
\hline & 6 & 108,0 & 11,8 & 507 & 1,87 & 1,05 \\
\hline & 7 & 138,8 & 3,3 & 653 & 1,46 & 0,85 \\
\hline & Total & 102,6 & 22,8 & 3266 & 2,17 & 1,33 \\
\hline & 1 & 125,6 & 29,7 & 593 & 1.98 & 0,95 \\
\hline & 2 & 84,4 & 9,4 & 350 & 3,36 & 1,41 \\
\hline & 3 & 115,2 & 11,3 & 514 & 2,01 & 1,14 \\
\hline \multirow[t]{5}{*}{3} & 4 & 85,0 & 3,0 & 388 & 2,35 & 1,37 \\
\hline & 5 & 88,4 & 4,3 & 411 & 2,16 & 1,32 \\
\hline & 6 & 83,4 & 29,7 & 374 & 2,19 & 1,08 \\
\hline & 7 & 109,2 & 3,5 & 516 & 1,84 & 0,95 \\
\hline & Total & 98,7 & 23,2 & 3146 & 2,21 & 1,24 \\
\hline & & & & - & & \\
\hline
\end{tabular}


La grande ressemblance des mesures recueillies sur les 3 passages nous a amenée à considérer, pour chaque variable, une seule valeur correspondant à sa moyenne calculée sur les trois passages. Le logiciel de calcul utilisé a corrigé en même temps les largeurs en fonction de l'angle d'inclinaison des cernes.

Ces différentes opérations nous ont finalement fourni des informations sur les caractéristiques microdensitométriques et la structure de 8793 cernes.

Le tableau I présente notre échantillon du point de vue de l'âge des arbres sondés, le nombre de cernes mesurés et leur largeur, par placette et type de station.

\section{RÉSULTATS}

Variabilité et liaisons entre les caractéristiques du bois mesurées

Les figures 3 et 4 illustrent la distribution des variables mesurées sur les 8793 cernes.
L'ensemble des variables présente une très grande dispersion, à l'exception de la largeur du bois initial (LBI) pour laquelle la variabilité apparaît beaucoup plus modérée.

Les distributions de la largeur de cerne (LC) et de ses composantes (LBI et LBF) montrent une nette dissymétrie, avec des valeurs extrêmes supérieures très dispersées. La largeur moyenne des cernes mesurés $(2,45 \mathrm{~mm})$ apparaît relativement élevée comparativement à ce que l'on peut trouver dans la bibliographie où les largeurs de cernes sont généralement comprises entre 1,5 et $2 \mathrm{~mm}$ (Polge et Keller, 1973 ; DeretVarcin, 1983 ; Zhang et al, 1993) mais où il s'agit souvent d'un mélange de chênes sessiles et de chênes pédonculés qui sont dans la majorité des cas plus âgés comparativement à notre échantillon.

Les composantes intracernes de la densité du bois suivent une distribution plutôt symétrique. La moyenne de la densité moyenne des cernes $\left(765 \mathrm{~g} / \mathrm{dm}^{3}\right)$ peut également sembler un peu élevée par rapport

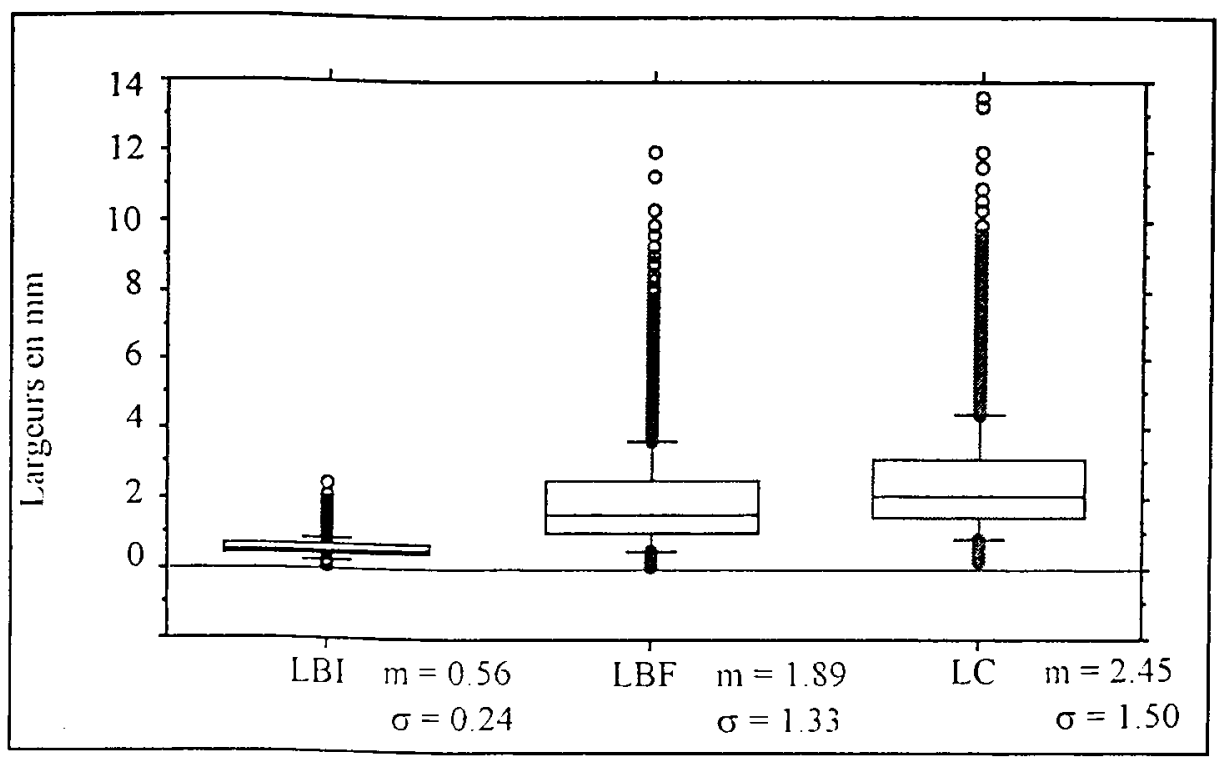

Fig 3. Distribution de la largeur du bois initial (LBI), de la largeur du bois final (LBF) et de la largeur de cerne (LC), mesurées sur les 8793 cernes étudiés. 


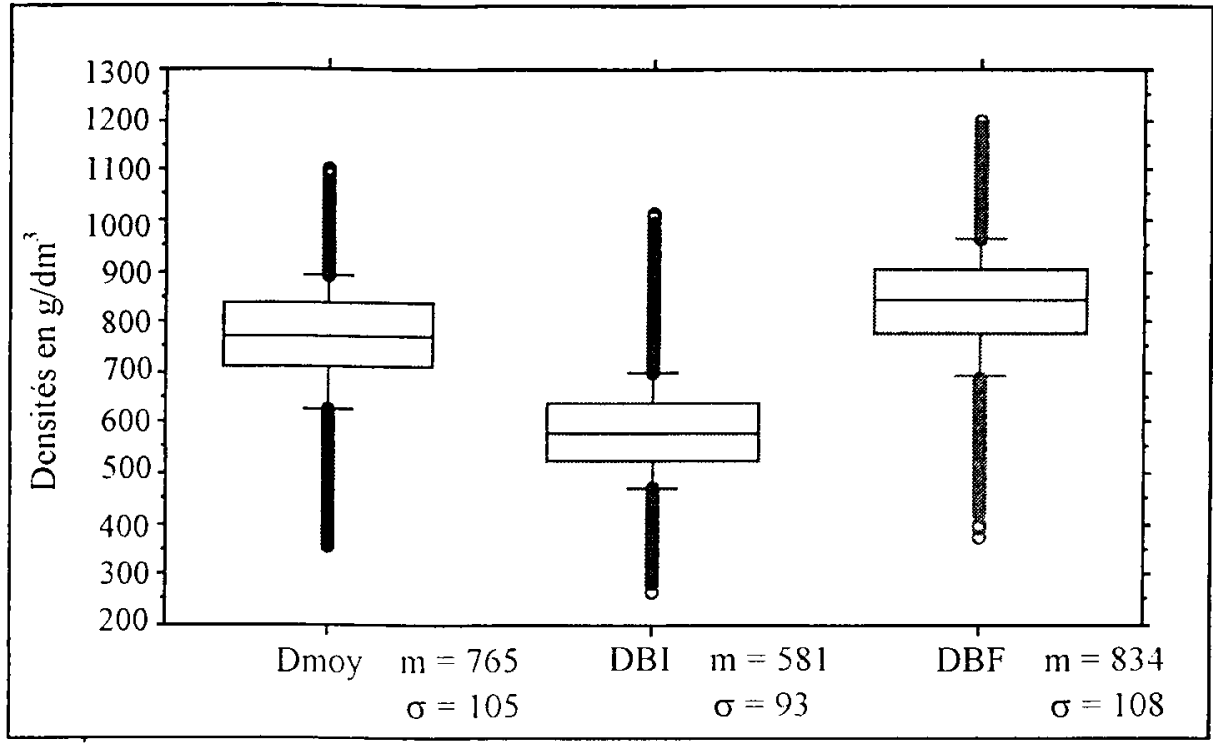

Fig 4. Distribution de la densité moyenne (Dmoy), de la densité du bois initial (DBI) et de la densité du bois final (DBF) mesurées sur les 8793 cernes étudiés.

aux $660 \mathrm{~g} / \mathrm{dm}^{3}$ trouvés par Zhang et al (1993) sur un échantillon de 23 chênes sessiles et pédonculés encore un fois plus âgés comparativement aux chênes pédonculés de notre échantillon.

Il existe d'importantes corrélations entre les diverses caractéristiques du bois, mesurées sur les 8793 cernes étudiés.

La largeur de cerne totale est corrélée positivement à ses composantes. Ainsi, la largeur du bois final est quasiment proportionnelle à la largeur totale du cerne $\left(R^{2}=\right.$ $0,98)$. La corrélation est moins forte avec la largeur du bois initial $\left(R^{2}=0,57\right)$ dont les valeurs sont de plus en plus dispersée lorsque la largeur de cerne augmente. $\mathrm{Ce}$ dernier point confirme les résultats d'Eyono Owoundi (1992) qui interprétait ce phénomène, sur un mélange de chênes sessiles et pédonculés, par l'existence d'une relation «largeur du bois initial - largeur du cerne totale" propre à chaque arbre.
La densité moyenne du cerne est également corrélée positivement à ses 2 composantes, en particulier à la densité du bois final $\left(R^{2}=0,92\right.$, vs un $R^{2}=0,66$ avec la densité du bois initial).

Les figures 5 et 6 montrent que la largeur de cerne et la densité moyenne diminuent toutes 2 graduellement lorsque l'âge cambial du cerne augmente, ce qui est en accord avec les résultats de Polge et Keller (1973) et d'Eyono Owoundi (1992). Néanmoins, il existe toujours des exceptions et l'on peut constater sur les figures 5 et 6 que les cernes les plus vieux de notre échantillon présentent, dans l'ensemble, des largeurs et des densités moyennes encore relativement élevées. Ces cernes appartiennent aux arbres de la placette 7 du type de station 2 (station neutrophile mésophile de coteaux).

Enfin, il n'existe pas de relation linéaire simple entre la largeur et la densité 
Fig 5. Influence de l'âge cambial du cerne sur la largeur de cerne (LC). Échantillon de 8793 cernes.

Fig 6. Influence de l'âge cambial du cerne sur la densité moyenne du cerne (Dmoy). Échantillon de 8793 cernes.
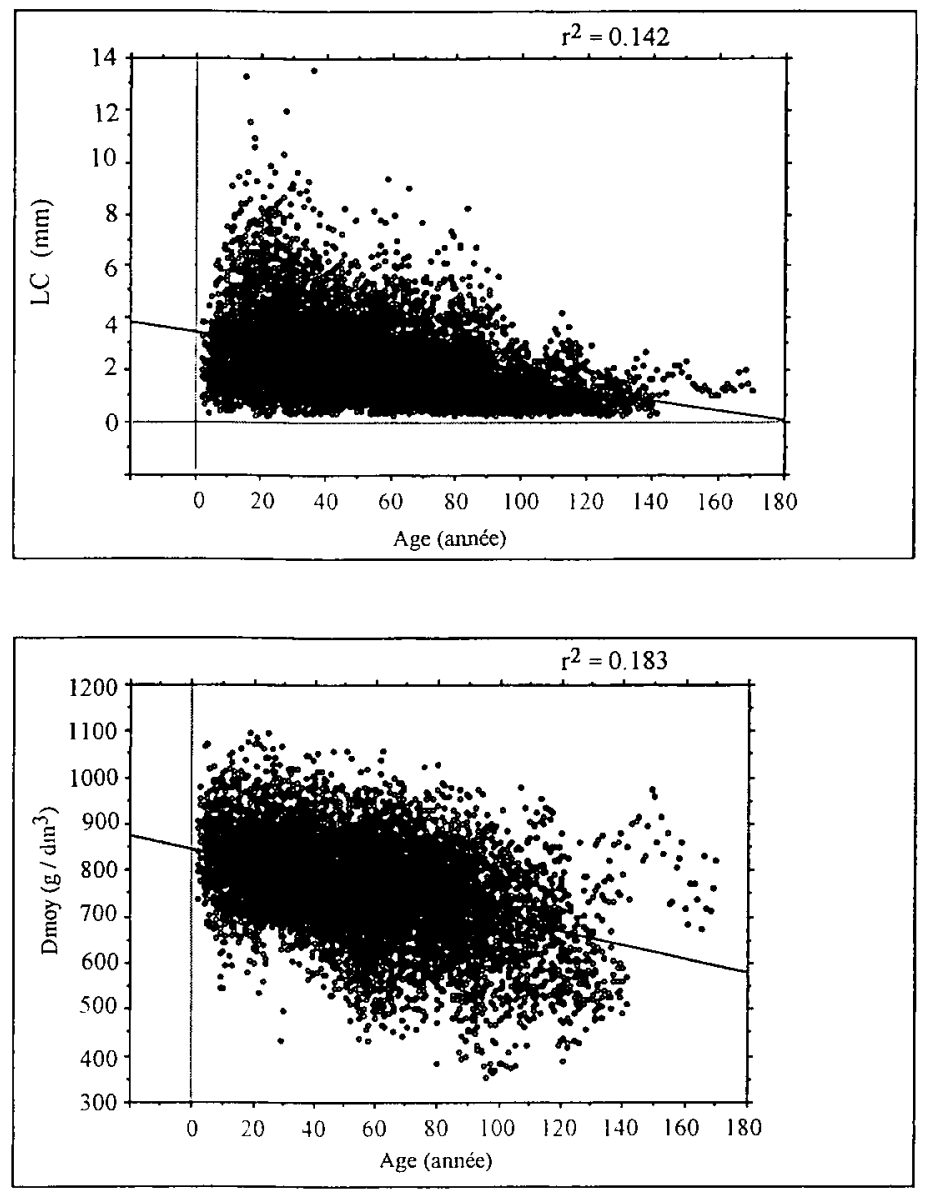

moyenne des cernes (fig 7). On note en particulier, comme l'avait signalé Eyono Owoundi (1992), une très forte dispersion des valeurs de la densité moyenne pour les largeurs de cernes les plus représentées dans notre échantillon ( 1,5 à $3,5 \mathrm{~mm}$ ).

\section{Influence du type de station sur les caractéristiques mesurées à âge de cerne fixé}

Connaissant l'influence de l'âge cambial des cernes sur la largeur des accroissements annuels et compte tenu de l'absence d'homogénéité des âges entre placettes et types de stations (tableau I), il est apparu indispensable de fixer artificiellement le facteur «âge cambial des cernes» pour analyser, sans biais inhérent à la largeur des cernes, l'influence des facteurs stationnels sur les composantes intracernes de la densité du bois.

Pour ce faire, nous avons sélectionné, dans notre échantillon de 8793 cernes, un sous-échantillon de cernes appartenant à une classe d'âge commune à tous les arbres étudiés. Pour avoir le même effectif de 


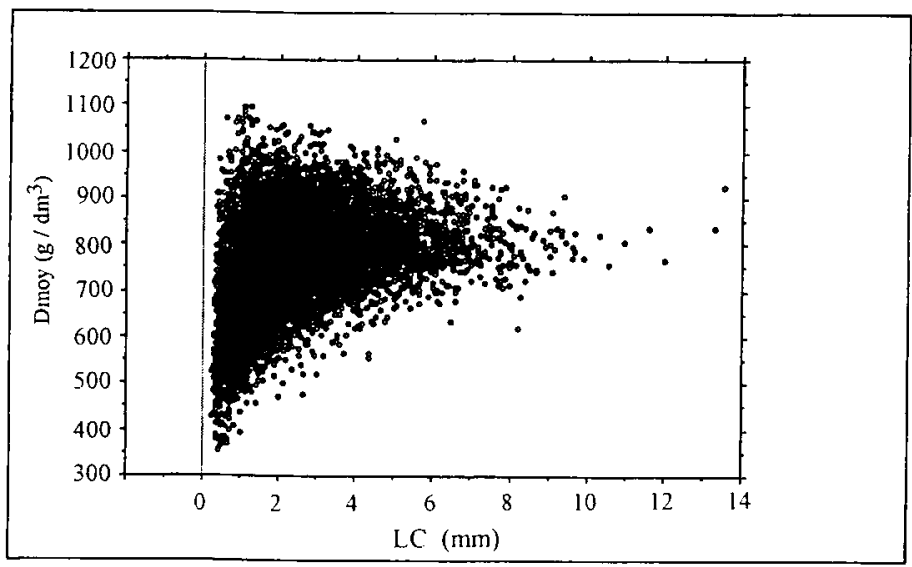

Fig 7. Relation entre la densité moyenne du cerne (Dmoy) et la largeur de cerne (LC). Échantillon de 8793 cernes.

cernes par arbre (possibilité de cernes manquants), nous avons pris en considération 35 cernes par arbre à partir du cerne de 20 ans. Ceci nous conduit à traiter un échantillon de 3675 cernes avec des effectifs égaux par arbre (35 cernes), par placette (175 cernes) et par type de station (1 225 cernes).

Sur ce nouvel échantillon, une analyse de variance suivant un modèle hiérarchique à 3 critères de classification (le type de station, la placette dans le type de station et l'arbre dans la placette) a été effectuée pour chacune des caractéristiques du bois mesurées.

Le tableau II présente les résultats de ces analyses de variances. 11 apparaît, dans un premier temps que, pour tous les paramètres mesurés, les 3 facteurs de variations considérés n'expliquent qu'une part restreinte de la variabilité totale observée. En effet, la variabilité résiduelle explique, à elle seule, 46 à $67 \%$ de la variabilité totale des paramètres mesurés. Elle correspond à la variabilité intercernes des paramètres à l'intérieur des arbres et reflète les variations climatiques interannuelles, le vieillissement physiologique de l'arbre (évolution de l'âge cambial des cernes) et l'impact des facteurs sylvicoles (en particulier les éclaircies).
C'est ensuite le facteur «arbre dans placette" qui explique la plus grande part de la variabilité restante. II est toujours significatif au seuil $1 \%$ o $\left(F_{(84,3570)}\right.$ compris entre $10,56^{\star * *}$ pour la largeur du bois initial et $25,56^{\star \star \star}$ pour la densité du bois final). Ceci confirme la très forte variabilité individuelle des propriétés du bois de chêne.

Le reste de la variabilité observée correspond à la variabilité inter-placette et à la variabilité inter-type de station. Suivant les caractéristiques du bois considérées, les facteurs «placette» et «type de station» se hiérarchisent différemment.

L'influence du type de station sur la densité moyenne (Dmoy) est significative au seuil de $1 \%$ et au seuil de $1 \%$ sur la densité du bois final (DBF).

Elle n'est significative qu'au seuil de 5\% sur la densité du bois initial (DBI), la largeur du bois initial (LBI) et la largeur de cerne totale (LC).

La largeur du bois final (LBF), quant à elle, n'apparaît pas significativement différente d'un type de station à l'autre. Elle dépend en revanche de la placette considérée dans chaque type de station. Cela tend à confirmer l'influence prépondérante de la sylviculture sur la formation de bois final (Polge et Keller, 1973). 
Type de station et qualité du bois de chêne

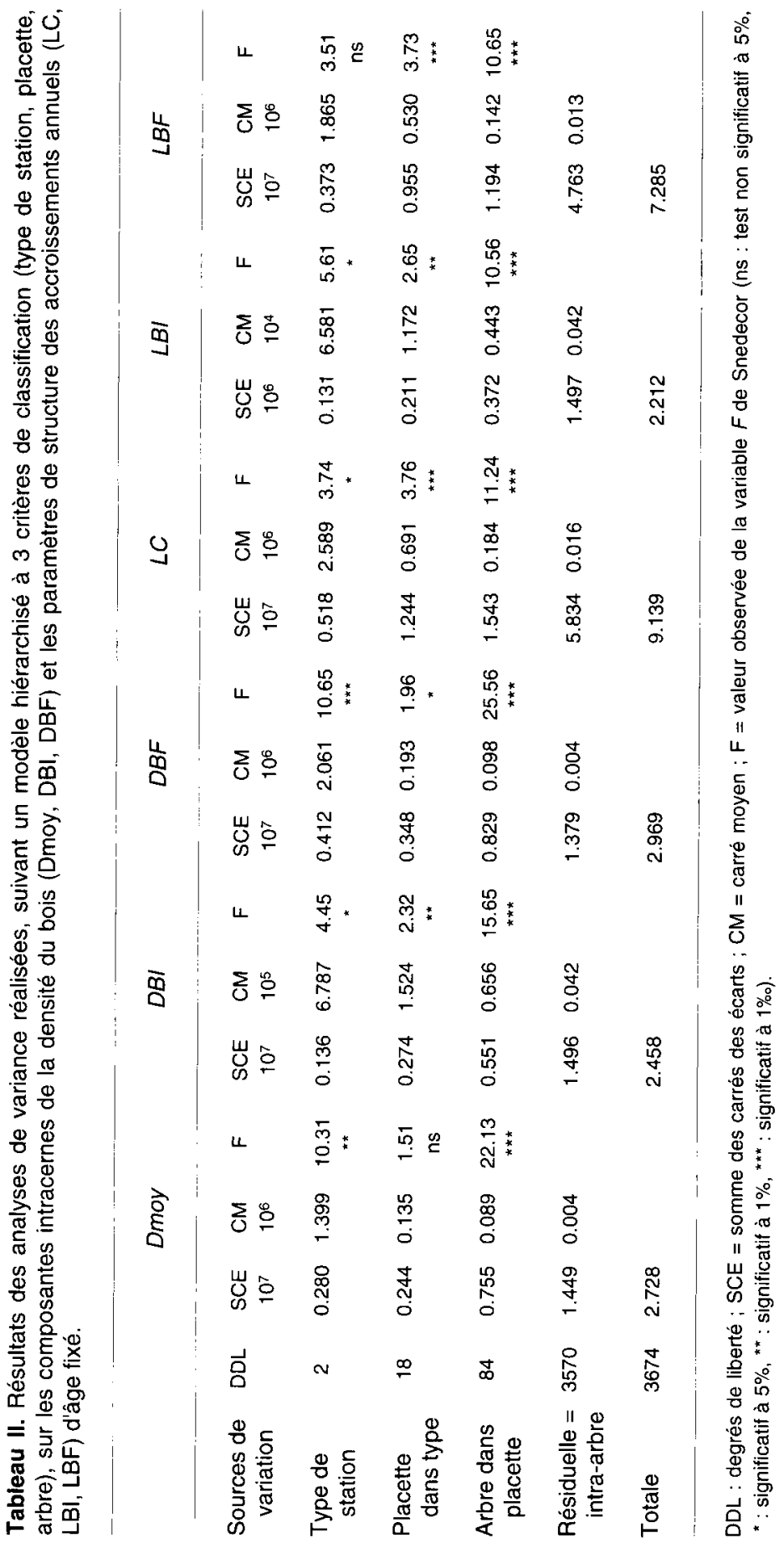


La figure 8 montre qu'en moyenne la densité moyenne des cernes augmente graduellement du type de station 1 (stations neutrophiles hygroclines de vallée) au type de station 3 (stations acidiphiles à acidiphiles modérées, mésophiles, de coteaux), lorsque les facteurs du milieu s'éloignent des conditions écologiques optimales de croissance du chêne pédonculé.

Néanmoins, cette augmentation de la densité moyenne des cernes ne peut être attribuée, dans l'immédiat, à une influence unique du type de station car les largeurs de cerne ne sont pas rigoureusement comparables entre les 3 types de stations (fig 9). En effet, le premier type de station, qui correspond à l'optimum écologique de l'essence sur les plans hydrique et trophique, présente des largeurs de cernes significativement supérieures à celles des 2 autres types de stations où les conditions hydriques sont défavorables à l'essence (vérifié par les tests de comparaison des moyennes 2 à 2 de Fisher et de Scheffé).

On peut en outre noter que la diminution du niveau trophique entre le type 2 et le type 3 , de même niveau hydrique, ne semble pas affecter la largeur des cernes qui reste sensiblement équivalente.
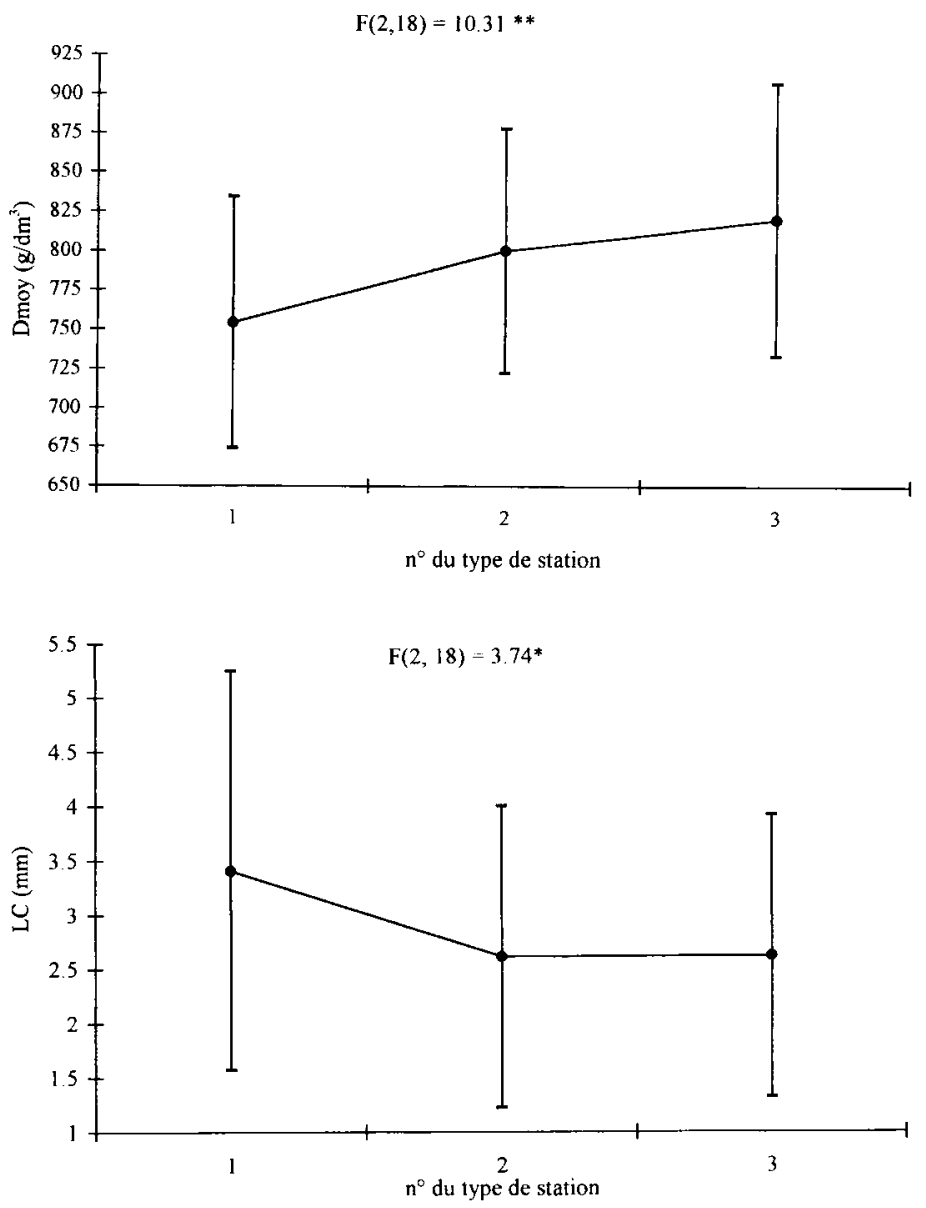

Fig 8. Influence du type de station sur la densité moyenne des cernes (Dmoy) à âge cambial fixé. Moyennes et écart types par type de station. Effectifs: 1225 cernes par type de station.
Fig 9. Influence du type de station sur la largeur des cernes (LC) à âge cambial fixé. Moyennes et écarts types par type de station. Effectifs : 1225 cernes par type de station. 
Le niveau hydrique des stations semble donc être le facteur stationnel majeur intervenant dans le déterminisme de la vitesse de croissance en diamètre du chêne pédonculé.

Ceci dit, pour ce qui est de l'influence stationnelle sur la densité moyenne des cernes, l'examen simultané des figures 8 et 9 montre que les types de stations 2 et 3 présentent des cernes de densités moyennes significativement différentes pour des largeurs de cernes sensiblement équivalentes. Le type de station pourrait donc bien avoir une influence sur la densité du bois qui ne passe pas par la vitesse de croissance.

Pour vérifier cette tendance, et pouvoir comparer la qualité du bois des arbres de nos 3 types de stations à travers les composantes intracernes de la densité du bois, toutes choses égales par ailleurs, nous avons fixé, en plus de l'âge des cernes, leur largeur.

\section{Influence du type de station sur les caractéristiques du bois mesurées, à âge et à largueur de cerne fixés}

Nous avons extrait, de l'échantillon précédent de cernes d'âge fixé, tous les cernes compris entre 1,5 et $3 \mathrm{~mm}$. Cette fourchette de largeur d'accroissement permet de représenter au mieux tous les arbres échantillonnés.

Nous noterons, cependant, que ce tri peut présenter l'inconvénient de considérer des années à faible croissance dans les bonnes stations et, au contraire, des années à croissance relativement forte dans les stations les plus mauvaises.

Notre nouvel échantillon comporte 1416 cernes (397 pour le type de station 1, 550 pour le type de station 2, 569 pour le type de station 3).
Comme précédemment, nous avons réalisé une analyse de variance suivant un modèle hiérarchique à 3 critères de classification (le type de station, la placette dans le type de station et l'arbre dans la placette) pour chacun des paramètres mesurés.

Les résultats de ces analyses sont présentés dans le tableau III. En premier lieu, nous pouvons vérifier que, tout en ayant fixé la largeur de cerne, l'âge des cernes reste comparable entre les 3 types de stations $\left(F_{(2,18)}=3,33\right.$ non significatif au seuil $5 \%$ ).

En ce qui concerne les paramètres de structure des cernes, nous constatons qu'en fixant la largeur de cerne nous avons également rendu invariable la largeur du bois final (LBF) entre placettes et types de stations $\left(F_{(18,84)}\right.$ et $F_{(2,18)}$ non significatifs au seuil $5 \%$ ). Ceci confirme la très forte corrélation mise en évidence entre la largeur de cerne et la largeur du bois final.

En revanche, la largeur du bois initial (LBI) apparaît significativement influencée par le type de station $\left(F_{(2,18)}=9,97^{\star \star}\right)$, malgré une très forte variabilité individuelle à l'intérieur des placettes $\left(F_{(84,114)}=4,28^{\star \star \star}\right)$ et une très grande variabilité résiduelle $(75 \%$ de la variabilité totale). En tout état de cause, les placettes d'un même type de station présentent des largeurs de bois initial relativement homogènes (variabilité inter-placette à l'intérieur du type de station non significative).

La figure 10, qui présente les moyennes et les écarts types de la largeur du bois initial dans les 3 types de stations, montre qu'en fait seul le type de station 1 (stations neutrophiles hygroclines de vallée) s'individualise des 2 autres types avec des largeurs de bois initial plus élevées (vérifié par les tests de comparaison des moyennes 2 à 2 de Fisher et de Scheffé).

La largeur du bois initial, à largeur d'accroissement et âge constants, serait donc essentiellement dépendante du niveau 


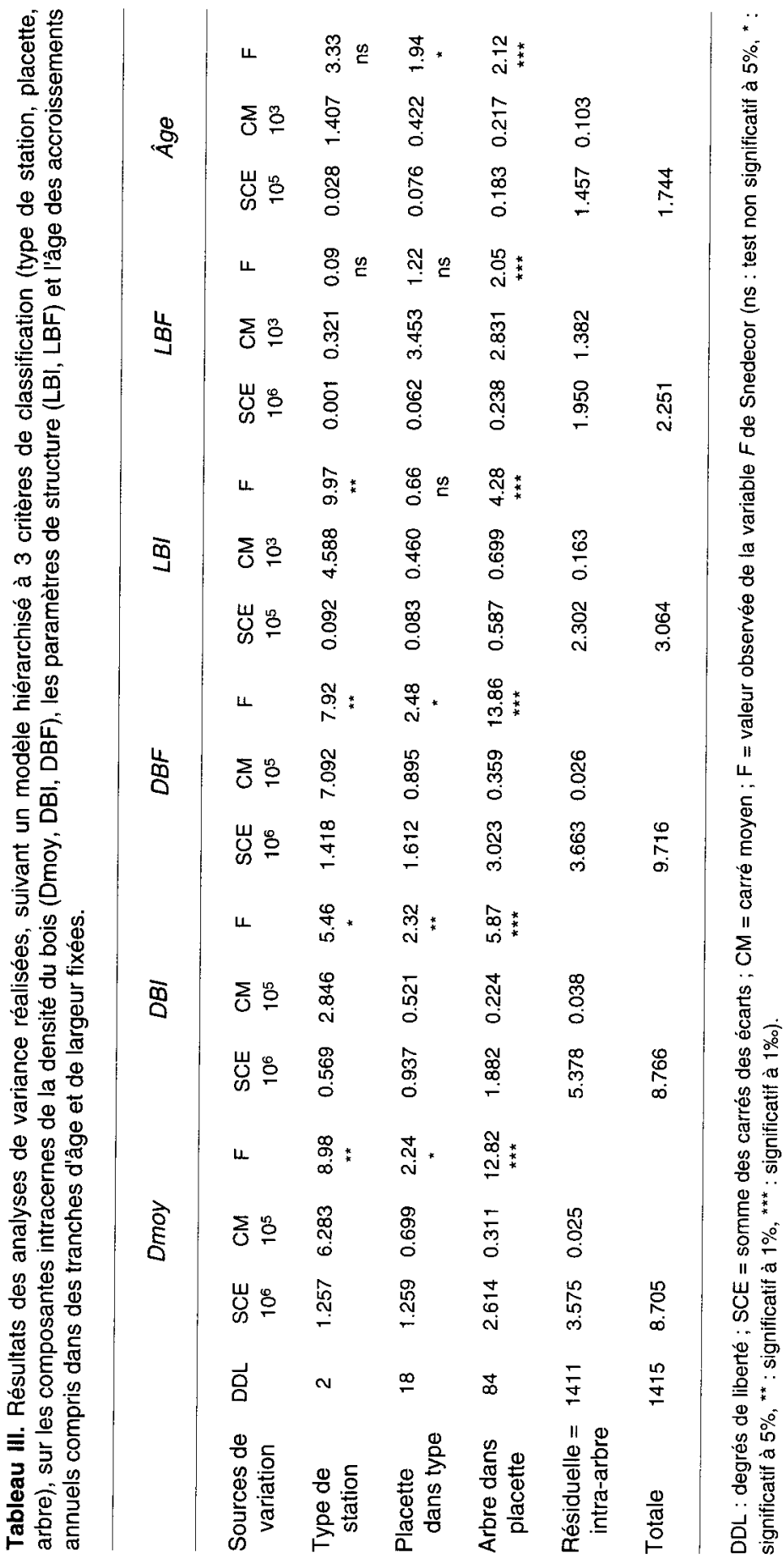


Fig 10. Influence du type de station sur la largeur du bois initial (LBI) à âge et largeur de cerne fixés. Moyennes et écarts types par type de station. Effectifs inégaux $(N 1=397 ; N 2=550$; N3 $=569$ ).

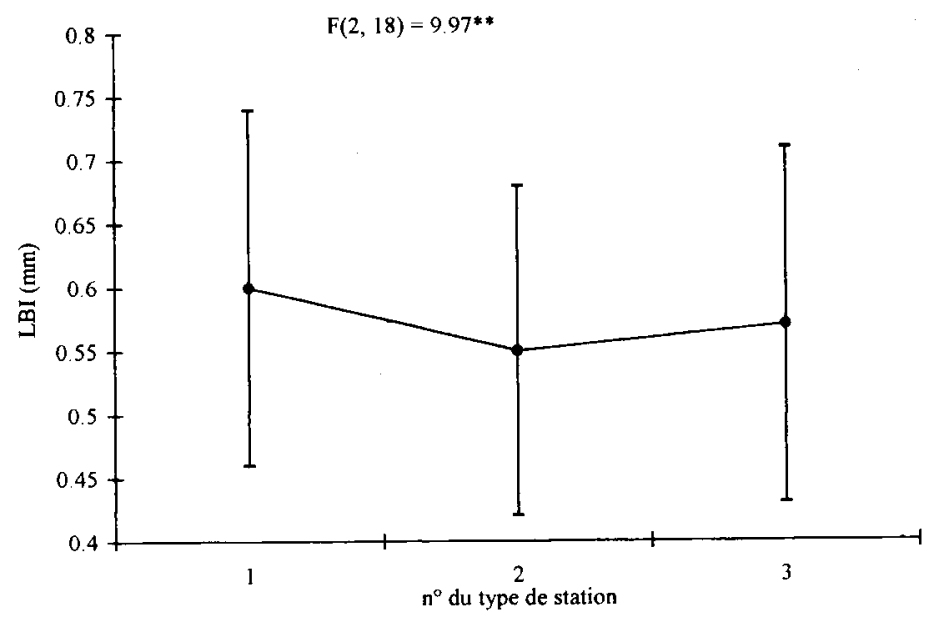

hydrique des stations. Ceci rappelle les résultats de Le Tacon et Mormiche (1974) où la largeur du bois initial, chez le chêne sessile (Quercus petraea), dépendait principalement de la réserve en eau.

Pour les 3 composantes intracernes de la densité du bois, la variabilité résiduelle reste très importante $(37,7 \%$ à $61,3 \%$ de la variabilité totale) et l'effet "arbre" apparaît toujours très hautement significatif $\left(F_{(84,1411)}\right.$ compris entre $21,5^{\star \star \star}$ pour la densité du bois initial et $31,1^{\star \star \star}$ pour la densité du bois final).

Néanmoins, l'influence du type de station sur la densité moyenne des cernes (Dmoy) se confirme $\left(F_{(2,18)}=8,98^{\star \star}\right)$ et il semble qu'elle s'exprime préférentieliement au niveau du bois final $\left(F_{(2,18)}=7,92^{\star \star}\right.$ pour la densité du bois final contre $5,46^{\star}$ pour la densité du bois initial).

Si l'on considère les moyennes par type de station de ces 3 composantes intracernes de la densité du bois (fig 11), il apparaît que plus on s'éloigne des exigences autécologiques du chêne pédonculé (du type 1 vers le type 3 ), plus ces dernières augmentent, donc plus les propriétés technologiques du bois auraient tendance à se dégrader.

\section{DISCUSSION}

Grâce à une approche méthodologique originale, reposant sur le contrôle à divers niveaux des différents facteurs agissant sur le déterminisme des propriétés du bois, il nous a été possible d'analyser indépendamment l'influence du type de station sur la qualité du bois du chêne pédonculé, estimée par les composantes intracernes de la densité du bois.

Malgré la très forte variabilité individuelle des caractéristiques mesurées (très fort effet «arbre»), il est apparu, à âge de cerne fixé, puis à âge et largeur de cerne fixés, que les composantes intracernes de la densité du bois, et en particulier la densité du bois final, étaient significativement différentes dans les 3 types de stations étudiés. Ces 3 caractéristiques augmentent, en effet, graduellement lorsque les facteurs du milieu s'écartent des exigences autoécologiques du chêne pédonculé (du type de station 1 au type de station 3), ce qui devrait se traduire par une diminution de la qualité du bois.

Le niveau hydrique des stations semble avoir une influence fondamentale sur la structure des accroissements annuels et 
sur les composantes intracernes de la densité du bois.

À âge de cerne comparable entre les 3 types de stations étudiés, les largeurs de cernes sont significativement supérieures dans les stations neutrophiles hygroclines de
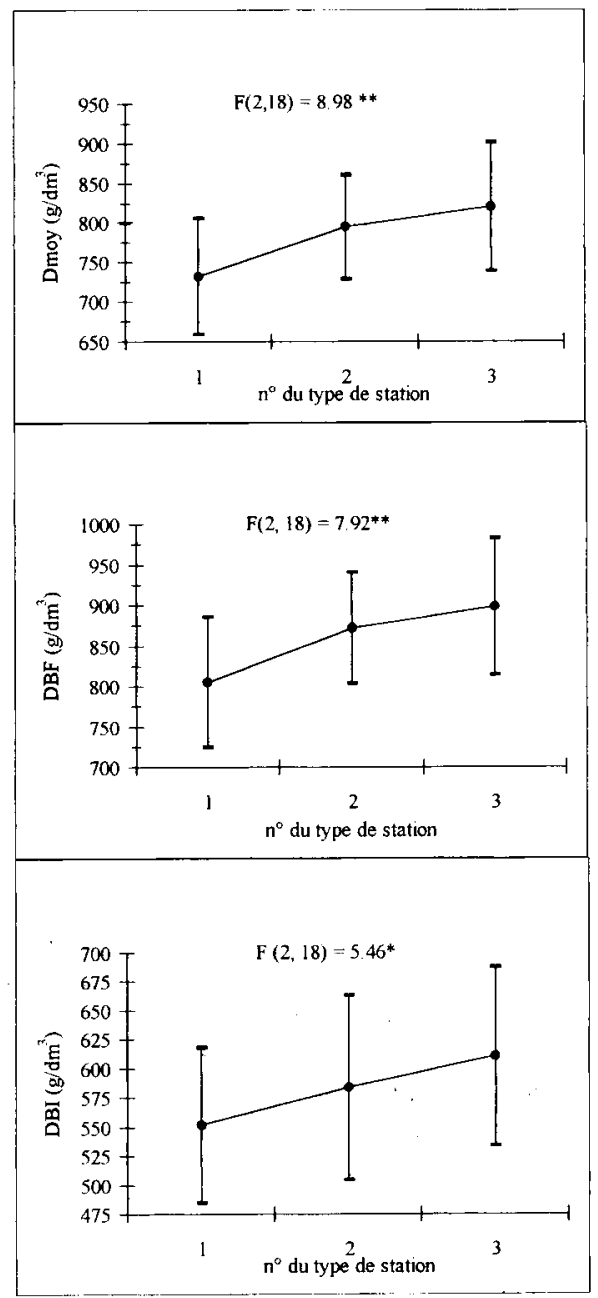

Fig 11. Influence du type de station sur les 3 composantes intracernes de la densité du bois (Dmoy, DBF, DBI) à âge et largeur de cerne fixés. Moyennes et écarts types par type de station. Effectifs inégaux ( $N 1=397 ; N 2=550$; $N 3=569$ ). vallée (type 1) comparativement aux stations neutrophiles à mésoneutrophiles et acidiphiles à acidiphiles modérées, mésophiles, de coteaux (types 2 et 3 ) qui présentent des vitesses de croissance en diamètre sensiblement équivalentes.

Ces stations neutrophiles hygroclines de vallée, offrant au chêne pédonculé des conditions écologiques optimales de croissance, présentent en outre les caractéristiques intracernes de densité les plus faibles.

Ceci rappelle les observations de Polge (1973) qui expliquait la faible densité du bois des chênes pédonculés de la vallée de la Saône, en dépit de leurs larges accroissements, par des conditions pédoclimatiques favorables à la formation dans le bois final d'une proportion de vaisseaux plus importante que la normale.

À âge et vitesse de croissance en diamètre comparables entre les 3 types de stations, les stations neutrophiles hygroclines de vallées se distinguent toujours par les composantes intracernes de la densité du bois les plus faibles et en plus par des largeurs de bois initial les plus importantes.

Le niveau trophique, pour les stations mésophiles de coteaux (type 2 et type 3 ), ne semble pas avoir d'influence sur la largeur des accroissements (échantillon de cernes d'âge fixé). En revanche il intervient lui aussi, à âge et largeur de cerne fixés, sur les composantes intracernes de la densité du bois qui augmentent lorsque le milieu s'acidifie (du type 2 au type 3).

Notre échantillonnage ne nous permet malheureusement pas de juger de l'influence du niveau trophique sur les caractéristiques intrinsèques du bois en milieu hygrocline de vallée (niveau hydrique correspondant à l'autécologie du chêne pédonculé).

Dans un premier temps, il serait donc intéressant d'élargir cette étude à un plus grand nombre de types de stations, en échantillonnant plus particulièrement plu- 
sieurs niveaux trophiques dans les milieux hygroclines de vallée.

Ensuite, pour une meilleure compréhension de l'influence des niveaux trophique et hydrique sur les propriétés du bois, il semble indispensable de passer à une échelle plus fine d'observation permettant d'apprécier l'influence de ces facteurs sur les éléments anatomiques constitutifs du plan ligneux.

Sur le plan pratique, les enseignements à tirer de cette étude pour la sylviculture du chêne pédonculé dans la région concernée ne sont pas négligeables.

Les stations neutrophiles hygroclines de vallée (type 1), qui correspondent aux exigences autécologiques du chêne pédonculé, présentent tous les atouts pour le maintien de la sylviculture de cette essence : vitesse de croissance en diamètre la plus élevée et meilleure qualité intrinsèque du bois observée.

En revanche, sur les coteaux, où les conditions hydriques sont moins favorables à l'essence, il ne semble pas raisonnable de maintenir le chêne pédonculé dans les stations les plus acides où les composantes intracernes de la densité du bois sont apparues les plus élevées (type 3). Sur les stations les plus riches chimiquement (type 2), il pourrait à la rigueur être maintenu mais dans les situations les plus fraîches.

Néanmoins, il serait souhaitable, pour appuyer ces propos, d'étudier l'impact technologique réel que peuvent avoir les variations des composantes intracernes de la densité du bois observées entre les 3 types de stations.

II faut également garder à l'esprit que la très forte variabilité individuelle des propriétés du bois chez le chêne pédonculé fait que le sylviculteur pourra toujours trouver des individus de médiocre qualité dans les stations a priori favorables à la production d'un bois de qualité et, inversement, trouver des individus de bonne qualité dans des milieux a priori peu propices à la récolte d'un bois de qualité.
Enfin, il reste aussi à considérer, en plus de critères de qualité interne, des critères de qualité externe (critères de forme et singularités). Ces derniers restent malheureusement encore très difficiles à aborder dans le cadre d'une étude des relations station-qualité du bois, en raison de leur caractère le plus souvent qualitatif et d'un manque de connaissance sur leur déterminisme.

\section{REMERCIEMENTS}

Ce travail n'a été rendu possible que grâce au concours des services départementaux de l'Office national des forêts des Landes et des PyrénéesAtlantiques qui nous ont autorisée à pratiquer des carottages à cœur et à $1,30 \mathrm{~m}$ sur les chênes choisis.

L'équipe de recherches sur la qualité des bois du centre INRA de Nancy a également joué un rôle considérable en mettant à notre disposition tout le matériel nécessaire à la réalisation des mesures. $G$ Nepveu et $F$ Mothe ont toute ma reconnaissance pour leur étroite collaboration aux cours des différentes phases de ce travail. Je tiens également à remercier Mme Garros pour son indispensable appui technique.

Enfin, je remercie très sincèrement $A$ Franc pour son soutien et ses nombreux conseils ainsi que JC Rameau, mon directeur de thèse.

\section{RÉFÉRENCES}

Ackermann F (1994a) Catalogue de types de stations forestières de la Chalosse, du Bas-Adour et des coteaux basques et béarnais. CEMAGREF, Groupement de Bordeaux, $507 p$

Ackermann $F$ (1994b) Étude de l'influence du type de station forestière sur la qualité du bois du chène pédonculé (Quercus robur $\mathrm{L}$ ) dans le sud-ouest de la France. Thèse de docteur en sciences forestières et sciences du bois, ENGREF Nancy, $156 p$ + annexes

Becker M (1979) Une étude phyto-écologique sur les plateaux calcaires du nord est (massif de Haye, 54). Utilisation de l'analyse factorielle des correspondances dans la typologie des stations. Relations entre la productivité et la qualité du hêtre et du chêne. Ann Sci For 36, 93-124 
Becker M, Le Tacon F, Timbal J (1980) Les plateaux calcaires de Lorraine. Types de stations et potentialités forestières. École nationale du génie rural, des eaux et des forêts, Nancy, $216 p+$ annexes

Courtoisier F (1976) Étude des relations entre station et qualité du bois de chêne en forêt de Bride et de Saint-Jean. Mémoire de $3^{e}$ année ENITEF, $48 p+$ annexes

Drapier J (1987) Étude de la potentialité forestière des stations par l'Inventaire forestier national. Les plateaux calcaires de haute Marne. Rev For Fr 39, 425435

Eyono Owoundi R (1992) Modélisation de la rétractabilité du bois en relation avec des paramètres de la structure de l'accroissement annuel et de la position dans l'arbre chez Quercus robur $L$ et $Q$ petraea Liebl. Application à l'intégration de la rétractabilité du bois dans les modèles de croissance de peuplements et d'arbres. Thèse de docteur en sciences du bois, ENGREF Nancy, $233 p$

Farrinotti S, Larousse JF (1991) Production et qualité des chênes en Sologne bourbonnaise : vers une optimalisation de la gestion des peuplements réguliers. Mémoire de $3^{e}$ année ENITEF, 114 p + annexes

Franc A, Curt T (1990) Étude des relations station-production pour le sapin pectiné dans les hautes Cévennes (Lozère). CEMAGREF, Riom, $30 \mathrm{p}$

Gilbert JM, Chevalier R, Vallée B (1991) Étude des relations station-production du douglas dans le pays d'Othe. Rapport interne CEMAGREF, division techniques forestières, Nogent-sur-Vernisson, $69 p$

Inventaire forestier national (1980) Département des Landes. Résultats du $2^{e}$ inventaire 1978, $\mathrm{t}$ l, doc IFN, Imp Nat, $79 \mathrm{p}$

Inventaire forestier national (1985) Département des Pyrénées-Atlantiques. Résultats du $2^{e}$ inventaire forestier, $\mathrm{t}$ I, doc IFN, Imp Nat, $211 \mathrm{p}$

Klumpers J (1990) La couleur du bois de chêne. Relation avec des facteurs sylvicoles environnementaux et individuels. Son incidence économique. DEA science du bois, univ Nancy I, $70 p+$ annexes

Le Goff N, Madesclaire A (1985) Étude de la potentialité des stations forestières des plateaux calcaires de Lorraine pour l'érable sycomore et le merisier. Colloque "Phytosociologie et foresterie", Nancy, 2022 novembre 1985

Le Tacon F, Mormiche A (1974) À propos de l'écologie du chêne de tranchage en Lorraine. Bull Techn ONF 6, 44-55

Madesciaire A (1986) Potentialité des stations forestières des plateaux caicaires de Lorraine pour l'érable sycomore et le meserier. Rapport d'étude CRPF Lorraine-Alsace/INRA Nancy

Mourey JM (1979) Les chênes de la vallée de l'Ognon (Franche-Comtè). Mémoire de $3^{e}$ année ENITEF, $252 \mathrm{p}$
Nepveu G (1988) Détermination de la qualité du bois par prélèvement non destructif. Rev For Fr 40, 62-70

Nepveu G (1989) Propositions pour l'étude des relations entre stations et qualité des bois. $I n$ : Station forestière, production et qualité des bois : éléments méthodologiques, CEMAGREF, groupement de Nogent-sur-Vernisson, 159-179

Nepveu $G$ (1990) Les facteurs influençant la qualité du bois de chêne (chêne rouvre et chêne pédonculé). Rev For Fr 42, 128-133

Nepveu G (1992) La qualité du bois de chêne (chêne rouvre et pédonculé) : définitions, sources de varia bilité, maîtrise possible. Travaux conduits par I'INRA ou en projet sur le sujet. Troisième journée professionnelle filière bois "L'aspect du bois de chêne et le classement», ENSAM, Cluny, 12 février 1992, 14 p

Nepveu G (1993) The possible status of wood quality in oak breeding programs (Quercus petraea liebl and Quercus robur L). Ann Sci For 50 (supp| 1), 388-394

Normandin D (1990) Le chêne français et ses produits dérivés. Marché intérieur et concurrence internationale. Rev For Fr 42, 110-118

Pellecuer B (1976) L'écologie des chênes en forêt de Morimond et ses applications sylvicoles. Thèse de docteur ingénieur, univ Nancy I, $173 p$

Perrin JR (1983) Dispositif de sciage de carotte de sondage de $5 \mathrm{~mm}$ sans collage préalable sur un support Ann Sci For 40, 399-406

Polge H (1966) Établissement des courbes de variation de la densité du bois par exploitation densitométrique de radiographies d'échantillons prélevés à la tarière sur des arbres vivants. Application dans les domaines technologique et physiologique. Ann SCi For 23, 1-206

Polge $\mathrm{H}$ (1973) Facteurs écologiques et qualitè du bois Ann Sci For 30, 307-328

Polge $H$ (1975) Étude préliminaire de l'influence de la fertilisation sur la qualité du bois de chêne. Rev For Fr 7, 201-208

Polge $H(1990)$ Chênes de marine et chênes de tranchage. Rev For Fr 42, 455-461

Polge H, Keller R (1973) Qualité du bois et largeur d'accroissements en forêt de Tronçais. Ann Sci For 30 , 91-126

Zahner R (1968) Site quality and wood quality in upland hard-woods: theoretical considerations of wood density. In : Tree Growth and Forest Soils, Oregon State University Press, ON, USA, 477-497

Zhang SY, Eyono Owoundi R, Nepveu G, Mothe F, Dhote JF (1993) Modelling wood density in European oak (Quercus petraea and Quercus robur) and simulating the silvicultural influence. Can J For Res 23, 2587-2593

Zhang SY, Nepveu G, Mothe F (1994) Modelling wood shrinkage in European oak by measuring wood density. For Prod J 40, 42-46 